\title{
Erratum to: The Method of Difference Potentials for the Helmholtz Equation Using Compact High Order Schemes
}

\author{
M. Medvinsky $\cdot$ S. Tsynkov $\cdot$ E. Turkel
}

(C) Springer Science+Business Media, LLC 2012

\section{Erratum to: J Sci Comput}

DOI 10.1007/s10915-012-9602-y

Funding information was omitted from the original publication. That information is as follows:

This work was supported by the US NSF under grant No. DMS-0810963, US-Israel Binational Science Foundation (BSF) under grant No. 2008094, and US ARO under grant No. W911NF-11-1-0384.

The online version of the original article can be found under doi:10.1007/s10915-012-9602-y.

M. Medvinsky $\cdot$ E. Turkel

School of Mathematical Sciences, Tel Aviv University, Ramat Aviv, Tel Aviv 69978, Israel

M. Medvinsky

e-mail: medvinsk@post.tau.ac.il

url: http://www.tau.ac.il/ medvinsk/

E. Turkel

e-mail: turkel@post.tau.ac.il

url: http://www.math.tau.ac.il/ turkel/

M. Medvinsky $\cdot$ S. Tsynkov $(\bowtie)$

Department of Mathematics, North Carolina State University, Box 8205, Raleigh, NC 27695, USA

e-mail: tsynkov@math.ncsu.edu

url: http://www.math.ncsu.edu/ stsynkov 